\title{
Evaluación de ruido urbano: el caso de la zona centro de Passo Fundo
}

Rodrigo Carlos Fritsch;

Rosa Maria Locatelli Kalil;

Eduardo Grala da Cunha

Filiación de autores:

FRITSCH, Rodrigo Carlos (Arquiteto e Urbanista, Msc. Professor contratado, Curso de Arquitetura e Urbanismo da Universidade de Passo Fundo) rcfritsch@upf.br

LOCATELLI KALIL, Rosa Maria (Dra.Arquiteto e Urbanista, Dr., Titular, Curso de Arquitetura e Urbanismo da Universidade de Passo Fundo) - kalil@upf.br

GRALA DA CUNHA, Eduardo (Dr.Arquiteto e Urbanista, Dr., Adjunto, Curso de Arquitetura e Urbanismo da Universidade de Passo Fundo) - egcunha@terra.com.br

\section{RESUMEN}

El presente estudio describe el resultado de la investigación, a nivel experimental, realizada en la zona centro de la ciudad de Passo Fundo, Rio Grande do Sul, Brasil; dicha investigación evalúa el nivel de polución sonora en las horas de mayor movimiento en cuatro puntos del centro de la ciudad. El estudio compara los datos obtenidos con la legislación municipal, con las normativas brasileñas vigentes y con las recomendaciones de las Organización Mundial de la Saludo (WHO).

Palabras-claves: POLUCIÓN SONORA; RUIDO URBANO; CONTAMINACIÓN SONORA.

\section{ABSTRACT}

The present study describes the result of a research in exploratory level that was carried out in a central area in Passo Fundo, Rio Grande do Sul, that evaluates the noise pollution state at rush diurnal schedules in four spots in this city. The study compares the data that were obtained with the town legislation and the brazilian standards in force and with the recommendations of the World Health Organization (WHO).

Key-words: NOISE POLLUTION; URBAN NOISE.

\section{I ntroducción}

El problema del ruido en la sociedad moderna viene disminuyendo gradualmente la calidad de vida de los ciudadanos de las grandes ciudades. Según Oliveira et al. (2000) "el ruido es una de las principales fuentes de perturbación en ambientes urbanos, y en general en las grandes ciudades representa el $70 \%$ de las reclamaciones a los órganos de control ambiental".

La preocupación por el control de los niveles de intensidad sonora en las grandes ciudades es relativamente reciente, es por este motivo que no existen estudios suficientes que nos sirvan como base teórica que nos auxilien en la toma de decisiones en la implantación de políticas mas efectivas para el control de la polución sonora. Según Niemeyer y Slama (1998) casi todo el conocimiento relativo al control de ruido urbano se realiza en países con un clima templado o frío, argumentan los autores que existe una gran carencia de estudios sistemáticos del ruido en ciudades de clima tropical, tanto en el ámbito legislativo como en las soluciones arquitectónicas. 
Las grandes ciudades europeas, y algunos centros urbanos de Brasil como Porto Alegre, Belo Horizonte, Curitiba y São Paulo, están poco a poco registrando sus áreas ruidosas, elaborando una base de datos que ciudades brasileñas de pequeño o medio tamaño todavía no poseen. Los estudios de ruido urbano son escasos, de momento, en Brasil, por lo que no podemos configurar una base de datos para enfrentar las necesidades de un planeamiento urbano integrado.

Este es el caso de la ciudad gaucha de Passo Fundo, localizada al norte del Estado de Rio Grande do Sul, en la que el problema de la contaminación sonora deteriora gradualmente la calidad de vida de sus habitantes, principalmente en el centro de la ciudad. La falta de datos urbanísticos de referencia ha sido un gran obstáculo para la investigación, ya que tan sólo desde ahora, en el año 2005/2006, el Ayuntamiento Municipal de Passo Fundo junto con la Universidad de Passo Fundo y la Universidad Federal de Rio Grande do Sul iniciaron un estudio serio respecto al comportamiento del tránsito en el centro de la ciudad, estudio importante para la evaluación del ruido del tráfico. Somos conscientes que para realizar una evaluación más rigurosa del ruido urbano necesitamos datos que hagan referencia a las características de flujo de vehículos como el porcentaje de vehículos pesados y ligeros, velocidad media, así como otros datos inexistentes hasta el momento.

En el presente trabajo las evaluaciones de ruido se realizaron teniendo en consideración los índices más usuales como el $\mathrm{L}_{e q} \mathrm{~dB}(\mathrm{~A})$ (Nivel equivalente de ruido en decibelios, ponderado en la sección A) así como, los índices estadísticos $L_{10}$ (Nivel de ruido de mayor actividad) y $L_{90}$ (Nivel de ruido de fondo).

Esta investigación, servirá como contribución y al mismo tiempo como marco inicial para el desarrollo de políticas más responsables de control de ruido en ciudades de tamaño medio como Passo Fundo.

\section{Contextualización}

El centro de Passo Fundo, viene sufriendo una fuerte masificación en las últimas décadas, estimulado por el Plan Director de Desarrollo Urbano de 1979 que estableció parámetros para el uso y la ocupación del suelo. El proceso se inició en la década de 1940 simultáneamente al proceso de expansión de las grandes capitales como Porto Alegre, Curitiba, Rio de Janeiro y São Paulo, entre otras, caracterizando el fenómeno conocido como metropolización.

Además del ordenamiento del crecimiento urbano, el Plan Director de 1979 tenía como objetivo contener la expansión dentro del área formada por las vías perimetrales Sur y Este, a través de la reglamentación del uso del suelo buscar una nueva imagen para la ciudad, que debería posicionarla como capital regional a nivel estadual.

Para ordenar el espacio físico-espacial de la ciudad de Passo Fundo, el plan del 1979 adopta una perspectiva basada en la industrialización, teniendo en consideración que Passo Fundo es un gran polo regional y un importante nudo de comunicaciones y de logística del interior del Estado para la distribución de las producciones de trigo y de soja y de productos importados. Esa postura busca una coherencia con el contexto de la expansión actual del cultivo de soja para otras regiones de Brasil, penetrando también en Argentina, Paraguay y Uruguay, previendo futuras relaciones incentivadas con el establecimiento del Mercosur. (GOSCH, 2002).

Con la consolidación del centro de Passo Fundo, los aspectos referentes al aumento de la densidad del área central empiezan a afectar directamente en la calidad de los espacios abiertos. La densidad y verticalización de la área construida ha provocado el sombreado permanente de las fachadas de algunos de los edificios en el centro 
de la ciudad, así como el aumento de la sensación de desconfort en función de los niveles elevados de ruido.

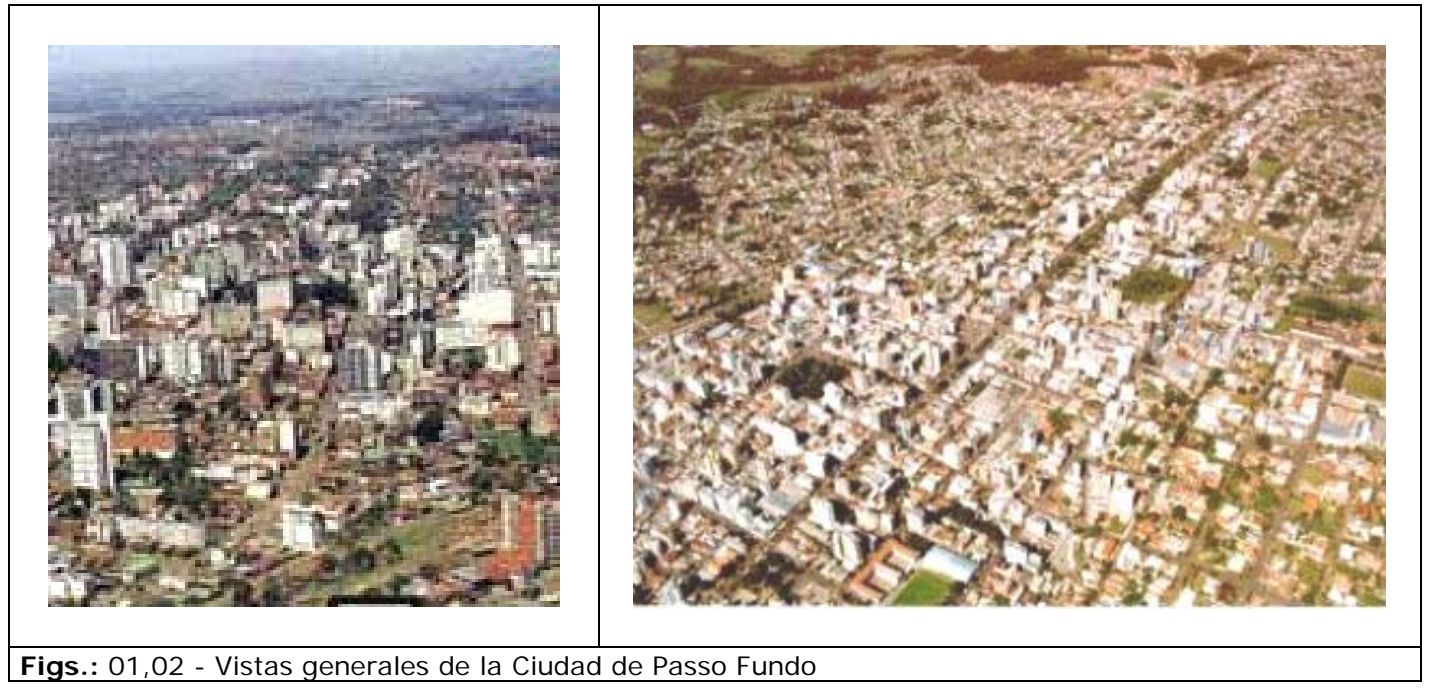

\section{Metodología}

Las mediciones experimentales de los niveles de ruido urbano se realizaron en cuatro puntos del centro de la ciudad en un día normal de la semana, concretamente un jueves. Se utilizó un medidor de presión sonora estándar del tipo 2, de la marca Instrutherm, recomendado por la NBR 10151 y la ANSI 2001, debidamente calibrado. Se monitorizaron las horas punta diurnas establecidos por la legislación de Passo Fundo siempre en la escala de compensación (A) y en el modo fast. Las franjas horarias de las mediciones fueron los siguientes: de las 07:00h a las 08:00h, de las 08:00h a las 09:00h, de las 11:00h a las 12:00h, de las $12: 00 \mathrm{~h}$ a las $13: 00 \mathrm{~h}$, de las $13: 00 \mathrm{~h}$ a las $14: 00 \mathrm{~h}$, de las $17: 00 \mathrm{~h}$ a las $18: 00 \mathrm{~h}$ y de las 18:00h a las 19:00h.

La monitorización se realizó por tanto durante 7 horas, con un total de 3600 datos por hora y 25.200 por día, mostrados cada 1 segundo. Para realizar la elección del día de la semana más adecuado para las mediciones se descartaron el lunes y el viernes por poseer una característica diferente de los otros días de la semana, ya que podríamos obtener valores atípicos en lo que se refiere a los niveles de ruido. Del mismo modo los niveles se alteran el sábado y el domingo, cuando los índices diurnos y nocturnos tienden a invertirse. Se determinó, que las mediciones se podrían llevar a cabo cualquier día entre el martes y el jueves que no fuese festivo, víspera de festivo o post-festivo. Así, en función de lo expuesto y por cuestiones de cronograma, se escogió el jueves para hacer la medición.

La elección de los puntos fue intencionada, basándonos en la importancia y en la sensibilidad al ruido de los equipamientos urbanos existentes en los locales como el Hospital de São Vicente de Paulo y el Hospital da Cidade, así como la importancia histórica de la Avenida Brasil y de la Calle Morom para la ciudad. Poseíamos información previa de que esos puntos sufren problemas en relación a niveles elevados de ruido, precisando tan sólo de una monitorización para evaluar el volumen de esos niveles, en realidad, ultrapasando los niveles permitidos y establecidos por la normativa vigente. Los datos referentes a las mediciones de los niveles de presión sonora se recogieron en locales donde el equipamiento pudiera ser montando sin problemas de interferencia de alguna barrera que involuntariamente, influenciase significativamente en el resultado de las 
mediciones. El montaje del equipamiento siguió las recomendaciones de la NBR 10151, obteniendo los siguiente parámetros:

- Valores horarios de $L_{e q}$;

- Valores horarios de $L_{\text {máx }}$ e $L_{\text {míni }}$

- $\quad$ Niveles estadísticos horarios de $\mathrm{L}_{10}$ y $\mathrm{L}_{90}$;

- Valores promedios para todo el día de Leq;

- Valores promedios para todo el día de $L_{10}$ y $L_{90}$.

Un dato importante a resaltar es que el sonómetro no facilitaba automáticamente los parámetros estadísticos $L_{10}$ y $L_{90}$, por lo que, los índices se estimaron conforme el procedimiento adoptado por DeBortolo (2000), a través de un histograma de frecuencias con un intervalo de clase de $5 \mathrm{~dB}$.

\section{Resultados}

A continuación, están presentados de modo resumido los resultados del día completo de las mediciones realizadas en los cuatro puntos de la ciudad. Las tablas siguen un padrón semejante al adoptado por Maia (2003), con las adaptaciones necesarias para la realidad metodológica de este trabajo. El montaje del equipo siguió el procedimiento establecido por la NBR 10151 con el sonómetro debidamente calibrado, en el modo fast con la ponderación en $\mathrm{dB}(\mathrm{A})$. Todas las mediciones se realizaron con buen tiempo, temperatura, humedad relativa y vientos del padrón necesario para una buena toma de datos.

Analizando los resultados obtenidos en función del $\mathrm{L}_{\mathrm{eq}} \mathrm{dB}(\mathrm{A})$ (Ver tabla 5), se constata que el punto más ruidoso entre los cuatro monitorizados es el Punto 1 , o sea, la Avenida Brasil con $73,2 \mathrm{~dB}(\mathrm{~A})$ de media, siendo el Punto 4 (Calle Teixeira Soares), lugar donde se encuentra el Hospital São Vicente de Paulo, un poco inferior, con un nivel medio de 71,6 dB(A). Esa diferencia en términos de presión sonora es significante, lo que lleva a la constatación de que ambos puntos están, prácticamente, con el mismo nivel de polución sonora. El tercer punto más ruidoso es el Punto 2 (Calle Morom) con 65,1 dB(A), prácticamente el mismo que el cuarto punto más ruidoso, el Punto 3 (Calle Paissandú) donde se encuentra el Hospital da Cidade, con $65,0 \mathrm{~dB}(\mathrm{~A})$. El nivel más elevado de ruido de actividad $\left(\mathrm{L}_{10}\right)$ también se da en el Punto 1 con $80,1 \mathrm{~dB}(A)$, así como el menor nivel de ruido de fondo ( $\left.L_{90}\right)$ se encontró en la Calle Teixeira Soares (Punto 4), donde se encuentra el Hospital São Vicente de Paulo con 56,7 dB(A).

En función de los resultados expuestos, podemos ahora, trazar algunos comentarios respecto a los niveles de ruido monitorizados en los cuatro puntos.

Tabla 1

Valores horarios diurnos del punto 1 (Avenida Brasil)

\begin{tabular}{|l|l|l|l|l|l|}
\hline Hora & $\begin{array}{l}\mathbf{L}_{\text {eq }} \\
(\mathbf{d B}(\mathbf{A}) \mathbf{)})\end{array}$ & $\mathbf{L}_{\text {mín }}$ & $\mathbf{L}_{\text {máx }}$ & $\mathbf{L}_{\mathbf{1 0}}$ & $\mathbf{L}_{\mathbf{9 0}}$ \\
\hline $07-08$ & 74,2 & 53,3 & 90,7 & 78,7 & 58,7 \\
\hline $08-09$ & 74,5 & 56,3 & 93,7 & 81,6 & 61,6 \\
\hline $11-12$ & 73,6 & 57,4 & 89,0 & 77,7 & 62,7 \\
\hline $12-13$ & 73,2 & 57,0 & 91,5 & 77,5 & 62,5 \\
\hline $13-14$ & 73,3 & 58,6 & 88,6 & 78,9 & 63,9 \\
\hline $17-18$ & 73,7 & 60,5 & 90,6 & 81,0 & 66,0 \\
\hline $18-19$ & 73,8 & 59,3 & 94,0 & 80,1 & 65,1 \\
\hline
\end{tabular}


Tabla 2

Valores horarios diurnos del punto 2 (Calle Moron)

\begin{tabular}{|l|l|l|l|l|l|}
\hline Hora & $\begin{array}{l}\mathbf{L}_{\text {eq }} \\
(\mathbf{d B}(\mathbf{A}))\end{array}$ & $\mathbf{L}_{\text {mín }}$ & $\mathbf{L}_{\text {máx }}$ & $\mathbf{L}_{\mathbf{1 0}}$ & $\mathbf{L}_{\mathbf{9 0}}$ \\
\hline $07-08$ & 62,9 & 47,4 & 84,9 & 67,9 & 52,9 \\
\hline $08-09$ & 63,5 & 51,1 & 82,6 & 66,1 & 56,1 \\
\hline $11-12$ & 64,3 & 53,1 & 84,0 & 68,5 & 58,5 \\
\hline $12-13$ & 62,5 & 51,2 & 83,0 & 66,3 & 56,3 \\
\hline $13-14$ & 62,9 & 52,4 & 80,5 & 67,5 & 57,5 \\
\hline $17-18$ & 64,4 & 52,9 & 88,1 & 68,0 & 58,0 \\
\hline $18-19$ & 66,1 & 55,9 & 89,8 & 70,9 & 60,9 \\
\hline
\end{tabular}

Tabla 3

- Valores horarios diurnos del punto 3 (Calle Paissandú)

\begin{tabular}{l|l|l|l|l|l}
\hline Hora & $\begin{array}{l}\mathbf{L}_{\text {eq }} \\
(\mathbf{d B}(\mathbf{A}))\end{array}$ & $\mathbf{L}_{\text {mín }}$ & $\mathbf{L}_{\text {máx }}$ & $\mathbf{L}_{\mathbf{1 0}}$ & $\mathbf{L}_{\mathbf{9 0}}$ \\
\hline $07-08$ & 65,4 & 48,4 & 88,1 & 68,7 & 53,7 \\
\hline $08-09$ & 66,8 & 52,4 & 89,9 & 68,0 & 58,0 \\
\hline $11-12$ & 64,4 & 50,0 & 83,1 & 70,2 & 55,2 \\
\hline $12-13$ & 63,2 & 48,6 & 82,0 & 68,6 & 53,6 \\
\hline $13-14$ & 63,8 & 50,7 & 81,1 & 65,7 & 55,7 \\
\hline $17-18$ & 65,0 & 52,5 & 86,3 & 67,8 & 57,8 \\
\hline $18-19$ & 65,4 & 52,0 & 88,6 & 67,5 & 57,5 \\
\hline
\end{tabular}

Tabla 4

- Valores horarios diurnos del punto 4 (Calle Teixeira Soares)

\begin{tabular}{|l|l|l|l|l|l|}
\hline Hora & $\begin{array}{l}\mathbf{L}_{\text {eq }} \\
(\mathbf{d B}(\mathbf{A}) \mathbf{)}\end{array}$ & $\mathbf{L}_{\text {mín }}$ & $\mathbf{L}_{\text {máx }}$ & $\mathbf{L}_{\mathbf{1 0}}$ & $\mathbf{L}_{\mathbf{9 0}}$ \\
\hline $07-08$ & 70,2 & 49,1 & 90,6 & 69,1 & 54,1 \\
\hline $08-09$ & 68,2 & 51,8 & 89,0 & 72,1 & 57,1 \\
\hline $11-12$ & 67,9 & 52,6 & 87,5 & 73,1 & 58,1 \\
\hline $12-13$ & 67,2 & 50,1 & 85,5 & 70,9 & 55,9 \\
\hline $13-14$ & 67,6 & 52,4 & 84,8 & 71,5 & 56,5 \\
\hline $17-18$ & 75,8 & 51,5 & 101,3 & 71,5 & 56,5 \\
\hline $18-19$ & 68,6 & 51,5 & 86,6 & 71,9 & 56,9 \\
\hline
\end{tabular}

Tabla 5

Comparación de los resultados diarios de los cuatro puntos monitorizados

\begin{tabular}{|c|c|c|c|c|}
\hline Puntos & $\mathbf{L}_{\mathbf{e q}}$ del día* & $\mathbf{L}_{\mathbf{1 0}}$ del día* & Lod del día* & Desvío Padrón \\
\hline Punto 1 - Avenida Brasil & 73,2 & 80,1 & 65,0 & 4,84 \\
\hline Punto 2 - Calle Moron & 65,1 & 69,6 & 59,6 & 4,04 \\
\hline Punto 3 - Calle Paissandú & 65,0 & 67,7 & 57,1 & 4,60 \\
\hline $\begin{array}{c}\text { Punto 4 - Calle Teixeira } \\
\text { Soares }\end{array}$ & 71,6 & 71,7 & 56,7 & 5,60 \\
\hline
\end{tabular}

*Todos los valores están expresados en $\mathrm{dB}(\mathrm{A})$. 


\subsection{I dentificación de las fuentes de mayor contribución de ruido}

De una manera general, podemos decir, que el tránsito es, sin duda alguna, el principal agente identificado en el proceso de polución sonora de la ciudad. El Ayuntamiento Municipal de Passo Fundo, a través del convenio firmado entre la Universidad Federal de Rio Grande do Sul - UFRGS y la Universidad de Passo Fundo - UPF, está realizando el primer estudio sobre los problemas en el tránsito de la ciudad. El estudio servirá para desarrollar el proyecto de implantación de la política municipal de tráfico, transporte y tránsito, pero especialmente en la Avenida Brasil, donde se constató previamente algunos problemas que están directamente unidos al aumento de la polución sonora en el área central SMSU (2005). Estos problemas, felizmente, son de fácil solución y comenzaron a ser sanados en enero de 2006. A continuación, serán catalogados algunos aspectos del tránsito que influyen directamente en la degradación del clima acústico en el centro de la ciudad de Passo Fundo.

\subsubsection{I nfluencia del tránsito en los niveles de ruido}

A través de la evaluación in loco y de las fuentes de archivo de la SMSU (2005), se pueden destacar tres factores determinantes para la degradación del clima acústico en el centro de la ciudad de Passo Fundo, siendo los siguientes:

1. Vehículos automotores: dependiendo de la concepción del proyecto de montaje de la estructura principal (chassis), de las condiciones de regulación, mantenimiento y la edad del vehículo, los niveles de ruido pueden llegar a picos de $90 \mathrm{~dB}$;

2. Semáforos no sincronizados: lo que hace que el tránsito pierda fluidez, obligando así al aumento en el número de frenadas y arrancadas por parte de los vehículos. Según la SMSU (2005), serán sustituidos por señalizadores electrónicos, así como de badenes físicos que serán sustituidos por limitadores de velocidad electrónicos;

Distancia entre las paradas de autobús: algunas de las paradas en el centro de la ciudad no están a más de 200 metros unas de las otras, obligando a los autobuses urbanos a realizar muchas frenadas y arrancadas, aumentando el nivel de incomodidad en función del ruido, además de dificultar la fluidez del tránsito en el centro de la ciudad.

Estos aspectos ratifican los resultados encontrados en la mediciones, percibimos que en los puntos donde encontramos los niveles de ruido más altos, también obtuvimos los mayores desvíos, poniendo en evidencia la oscilación de los niveles durante el día y la sensación de desconfort generada por esa variabilidad.

\subsubsection{Contribución de los vehículos automotores en relación a la polución sonora}

Durante la monitorización se realizó una recogida de datos buscando verificar de una manera más puntual la contribución individual de algunas de las fuentes de ruido, específicamente de vehículos automotores, en los puntos medidos. Se constató que los vehículos de mayor contribución para el aumento de los niveles de ruido en orden decreciente, son:

10 Vehículos pesados (autobuses y camiones): llegan a niveles de entre $80 \mathrm{~dB}(\mathrm{~A})$ y $85 \mathrm{~dB}(\mathrm{~A})$. Entre estos, los autobuses contribuyen más en la polución sonora en función de la mayor frecuencia en las calles, así como en el número de paradas y de arranques, aumentando la sensación de desconfort. 
Según Sousa (2004) los autobuses y camiones difieren de los vehículos leves en función del tipo de motor, movido por diesel, que impulsa a estos vehículos, aunque también se utilicen los de gasolina. La autora comenta que una característica importante que los diferencia es el proceso de combustión, haciendo que los camiones movidos por diesel tengan una mayor fluctuación de presión en los cilindros, resultando un mayor nivel de ruido. Otra característica, es que estos tipos de camiones alcanzan una velocidad media inferior, lo que determina una contribución mayor de ruido de baja frecuencia. Como dicho ruido de baja frecuencia se propaga a grandes distancias en el tejido urbano, existe la necesidad de la reglamentación de esos vehículos en la ciudad, principalmente durante la noche. En Brasil los autobuses se fabrican sobre chassis de camiones, haciendo que sean tan ruidosos como estos. Otro aspecto importante es la edad de la flota, cuanto más vieja sea mayores serán los niveles de ruido emitidos por ella.

2o - Vehículos leves que se dividen en:

a) Motocicletas: emiten niveles de ruido que varían en función de las clases de estos vehículos. El mayor problema reside en los tubos de escape abiertos sin silenciador, práctica bastante común entre los jóvenes, llegando a niveles entre 80 y $90 \mathrm{~dB}(\mathrm{~A})$ de media;

b) Utilitarios: con motores no regulados y con música, ciertamente son agentes que contaminan, mientras que los coches usados para publicidad itinerante tienen una contribución, actualmente en la ciudad, muy importante en la degradación del clima acústico del centro. Los niveles de música de esos coches en las mediciones llegaron a picos de $94 \mathrm{~dB}(\mathrm{~A})$ lo que provoca reacciones de gran incomodidad aparente en los transeúntes que desafortunadamente se encuentran la trayectoria del coche publicitario.

Del mismo modo, es importante anotar que los niveles de ruido en función de los coches publicitarios aumentan en la primera quincena del mes, cuando la población cobra sus sueldos y consecuentemente el ciudadano posee mayores condiciones de gasto. Las tiendas ponen estos vehículos con más frecuencia en este periodo contribuyendo en gran medida a la degradación de calidad de ambiente sonoro en el centro de Passo Fundo.

\subsubsection{Otras fuentes relevantes}

Otras fuentes identificadas alrededor a los puntos de medición fueron las tiendas direccionadas al público más popular. Ellas utilizan equipos de música con los altavoces orientados hacia la calle, donde el nivel de ruido medio, fue compatible muchas veces con el de los autobuses y camiones, ultrapasando en determinadas situaciones los $80 \mathrm{~dB}(\mathrm{~A})$. Los comerciantes optan por este tipo de soluciones, ya que el elevado nivel de ruido producido por el tráfico de vehículos inhibe las diferentes opciones que tienen los empresarios para llamar la atención de sus clientes potenciales, este aspecto genera una mayor degeneración acústica en el centro de la ciudad. 


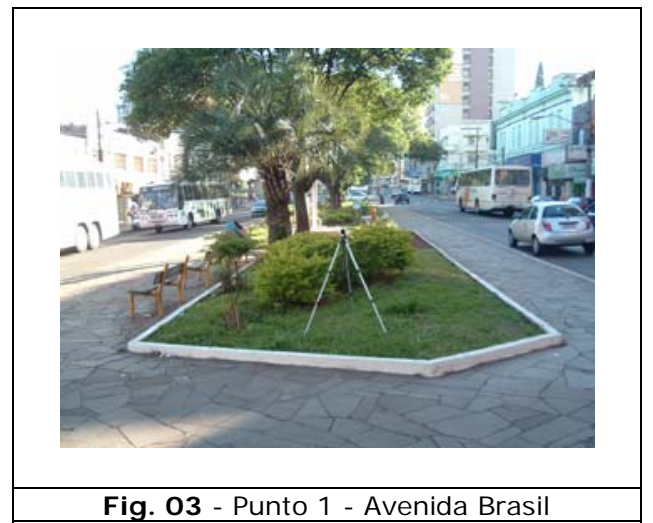

Fig. 03 - Punto 1 - Avenida Brasil
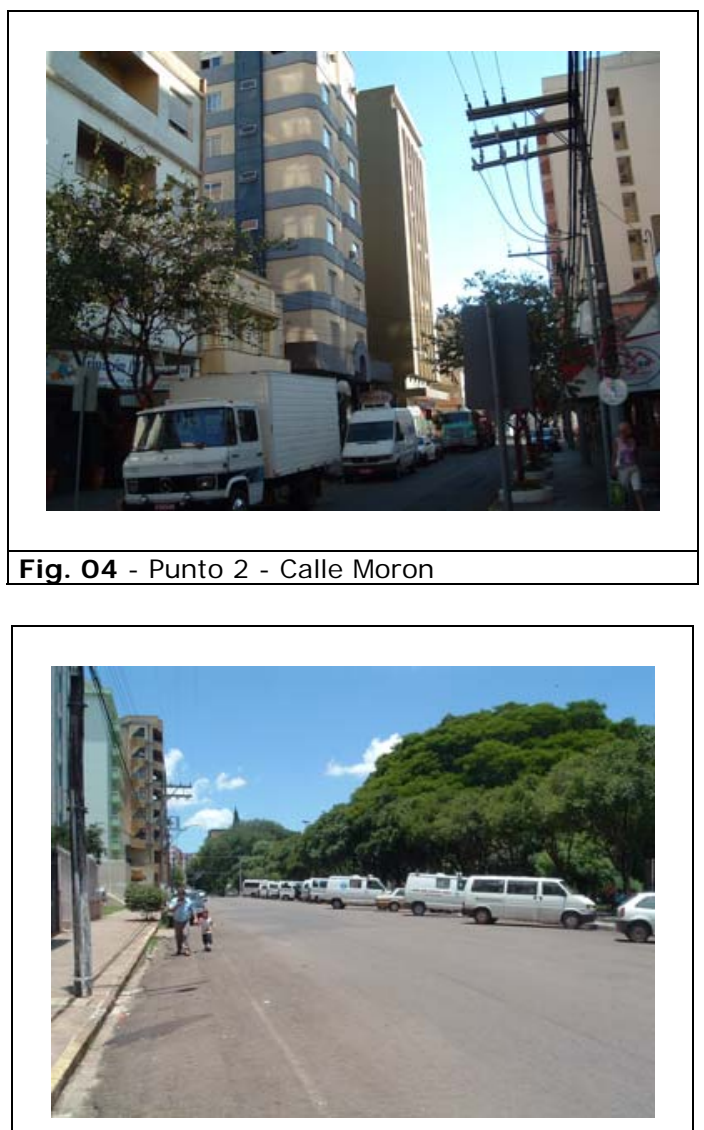

Fig.05- Punto 3 - Calle Paysandú 


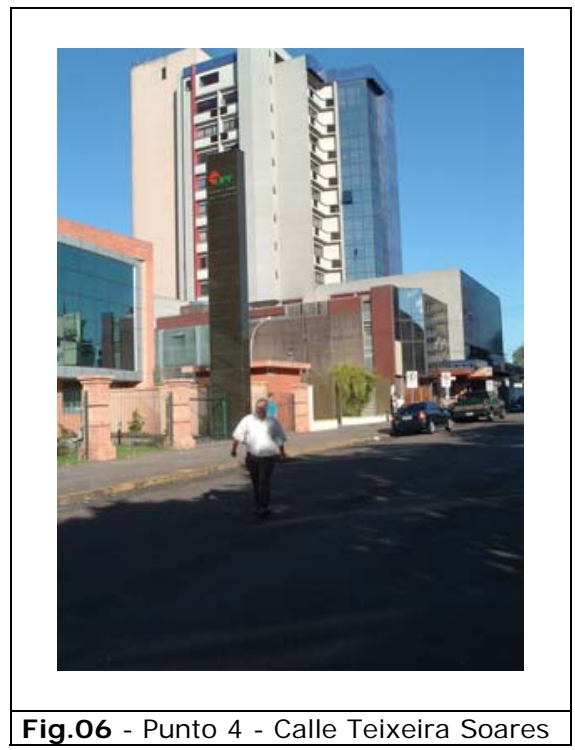

\subsection{Contribución del entorno}

Los análisis del entorno demuestran una homogeneidad respecto a los elementos compositivos del medio urbano en los cuatro puntos monitorizados. Se puede afirmar que, básicamente, los espacios estudiados poseen poca calidad acústica en función de 5 aspectos descritos a continuación.

\subsubsection{Aspectos relevantes referentes a calidad acústica del entorno}

1. Materiales utilizados en las calles y en los paseos poco absorbentes, promoviendo un mayor número de reflexiones sonoras, aumentando de este modo el tiempo de permanencia de la música en el aire. El revestimiento del asfalto se puede considerar semi-absorbente debido a su rugosidad, pero funciona de una manera más efectiva en el control del ruido absorbiendo la fricción de los neumáticos de los vehículos. Sabemos que una calle de paralelepípedos se vuelve mucho más ruidosa en este aspecto que en las vías revestidas de materiales con una base de alquitrán como el asfalto;

2. Vegetación poco representativa en lo que se refiere a una posible influencia sobre la atenuación del ruido en los puntos analizados;

3. Forma de las construcciones, sus separaciones frontales y las dimensiones de los edificios acaban siendo determinantes para una mala calidad del campo sonoro en los puntos estudiados, por lo que condicionan que el ruido permanezca más tiempo reverberando en el espacio, aumentando la sensación de incomodidad;

4. El conjunto de edificios acaba condicionando estas características constructivas que favorecen el aumento de la reverberación entre los mismos, manteniendo el ruido por más tiempo en el espacio. Del mismo modo, los materiales usados en las fachadas son, en su gran mayoría, reflexivos, no facilitan la absorción necesaria para un buen equilibrio acústico; 
5. Inexistencia de jerarquización aparente del tránsito en las vías estudiadas. Salvo en la Calle Moron, que no permite el tráfico de autobuses y camiones más pesados, las otras vías soportan la circulación de, prácticamente, todos los tipos de vehículos.

\subsubsection{La influencia de los índices urbanísticos}

Los parámetros como: la tasa de ocupación, el tamaño y las separaciones laterales y frontales que condicionan la implantación de la edificación en el solar, influencian en la forma del tejido urbano alterando la propiedad del campo sonoro generado por la fuente (NIEMEYER \& SLAMA, 1999). La característica lineal de los terrenos y de los bloques en el centro de la ciudad es un ejemplo claro de este aspecto.

En Passo Fundo la mayor tasa de ocupación para usos conformes (el que predomina en la zona, dándole la característica) es del $100 \%$ para los sótanos, planta baja y entresuelo, hasta una altura máxima de 7,00 m contando desde el peldaño de acceso hasta el techo del entresuelo. Dicho límite de altura podrá ser de tres plantas cuando sea para uso como garajes colectivos. Esta son las características de ocupación en la región donde está ubicado el Hospital da Cidade. Del mismo modo, existe un gran número de otros usos conforme donde se puede construir hasta el $100 \%$ del terreno, lo que acaba determinando la conformación lineal y alta de los edificios, referidos en los apartados anteriores.

La segunda mayor tasa de ocupación para uso conforme es del 75\%, está localizada en un área sensible, concretamente donde se encuentra el Hospital São Vicente de Paulo, lo que resulta una densificación muy elevada; se pueden constatar esas cuestiones analizando el Plan Director de Desarrollo Urbano de 1984.

Podemos concluir que, en la ocasión de los estudios para el desarrollo del Plan Director de 1984, no existió una preocupación por jerarquizar los usos en función de las actividades ruidosas, lo que en realidad, no era una práctica común en la mayor parte de países del mundo en aquella época y muchos menos en Brasil.

\section{Conclusiones}

El trabajo realizado constató que la degradación sonora en el centro de Passo Fundo ya está presente, encontrándose los niveles de ruido urbano por encima de los índices recomendados por la NBR10151 y la NBR 10152, así como los valores recomendados por la Organización Mundial de la Salud - OMS (WHO, 1980). En las zonas menos ruidosas el $L_{E Q}$ del día ya sobrepasa el máximo recomendable para los tipos de área monitorizados. Los valores de ruido de fondo ( $\left.L_{90}\right)$ apuntan claramente para esta cuestión, si tenemos en cuenta que los resultados en los cuatro puntos están por encima de $55 \mathrm{~dB}(\mathrm{~A})$ recomendados por la OMS (WHO, 1980). Podemos asociar el ruido del tráfico como agente directo en la degradación sonora existente hoy en el centro de la ciudad. Aún sabiendo que los datos monitorizados se tomaron en horas punta, los resultados se mostraron satisfactorios en lo que se refiere a la caracterización de un día típico de la semana de la ciudad para abarcar un número relevante de horas de contraste frente a los horarios punta, o sea, aquella horas inmediatamente anteriores y posteriores a los horarios más ruidosos, como, desde las 07 a las 08h de la mañana o el medio día, por ejemplo.

Otro factor importante a tener en cuenta es el hecho de que la Legislación Municipal referente al control de ruido, a través de la Ley 2.427 , posee niveles máximos permitidos por encima de las normas vigentes, tanto a nivel nacional como internacional, siendo necesaria una revisión en este sentido. 
La falta de una política clara de control del ruido en la ciudad de Passo Fundo requiere algunas recomendaciones como el desarrollo urbano del municipio. Estas recomendaciones son genéricas, pasando por cuestiones urbanas, constructivas y educacionales, que pueden ser aplicadas para ciudades con las mismas características, adaptándolas a la realidad de cada municipio. Las recomendaciones se dividen en acciones de control de corto, medio y largo plazo, descritas a continuación:

a) Acciones a corto plazo:

Abordar la cuestión de la polución sonora en Passo Fundo como un problema de salud pública;

Incluir el tema en las discusiones del nuevo Plan Director de Desarrollo Integrado de la ciudad;

Realizar una revisión de la legislación Municipal en lo que se refiere a los parámetros acústicos vigentes, para adecuarlos a parámetros que tengan en consideración la salud y el bien estar de la población;

Capacitar al cuerpo técnico de los órganos públicos y adquirir equipamientos que estén dentro de los patrones internacionales para la instrumentación adecuada de trabajo;

Jerarquizar efectivamente las vías con la ordenación del transito, desviando los vehículos más pesados de los alrededores de las áreas más sensibles de la ciudad como el Hospital São Vicente de Paulo y el Hospital da Cidade;

Fiscalizar de una manera más efectiva los establecimientos que no estén cumpliendo las normas vigentes, así como el tránsito, en lo que se refiere a niveles abusivos de música en coches particulares y publicitarios;

Promover campañas educacionales relativas a la polución sonora en las escuelas y para la población en general;

b) Acciones a medio y largo plazo:

Establecer una monitorización constante de ruido en la ciudad, en diversos puntos, las $24 \mathrm{~h}$ del día, para obtener un informe más completo de los niveles de presión sonora. Una buena solución para esto sería la instalación de relojes acústicos Relacus, iguales a los existentes en Curitiba - Paraná;

Confeccionar un mapa de ruidos para auxiliar la búsqueda de soluciones urbanísticas a nivel municipal, sirviendo de herramienta de planeamiento;

Iniciar la construcción de un banco de datos urbanísticos, siendo actualizado constantemente, comprendiendo estudios que abarquen las diferentes áreas de conocimiento dentro del ambiente urbano. Ese banco serviría como subsidio para la toma de decisiones por parte del poder público;

Realizar un estudio con criterio del medio urbano con la jerarquización de los usos en función de las fuentes de ruido;

Establecer colaboraciones entre el Ayuntamiento Municipal y la Universidad para el desarrollo de investigaciones relativas al control más efectivo del ruido en la ciudad. Esas recomendaciones son de gran importancia para la contribución en el desarrollo de políticas públicas que sean capaces de minimizar los impactos de crecimiento desordenado de las ciudades en la vida de los ciudadanos. Del mismo modo es importante destacar que las acciones deben ser ejecutadas por cuerpos multidisciplinares constituidos por profesionales especializados, lo que proporciona un mayor campo de investigación, así como una mayor capacidad de éxito en las acciones implantadas.

\section{Referencias bibliográficas}

NBR-1015: Acústica-Avaliação do ruído em áreas habitadas visando o conforto da comunidade-Procedimento, Rio de Janeiro: ABNT, 2000. 
NBR-10152: Acústica-Avaliação do ruído em recintos de edificações visando o conforto dos usuários - Procedimento, Rio de Janeiro: ABNT, 1987.

ANSI, 2001- American National Standard Institute, Specification of Sound Level Meters - ANSI S1.4-1983 (R 2001) with Amd.S1.4A-1985.

DE BORTOLI, Paulo Sérgio, Análise da Poluição Sonora Urbana em Zoneamentos Distintos da Cidade de Curitiba, Dissertação de mestrado, PPGTE/CEFET, Paraná, 2002.

GOSCH, Luiz Roberto Medeiros, Passo Fundo: De Saturnino de Brito ao Mercosul, Dissertação de Mestrado, Faculdade de Arquitetura e Urbanismo, UFRJ, Rio de Janeiro, 2002.

MAIA, Marco Antônio Lopes, Contribuição ao Mapeamento do Ruído Urbano na cidade de Porto Alegre, Dissertação de mestrado, PPGEC/UFRGS, Rio Grande do Sul, 2003.

NYEMEYER, M. L.; SANTOS, M. J. de Oliveira, Qualidade Acústica no Espaço Urbano, In: VI Encontro Nacional e III Encontro Latino-Americano sobre Conforto no Ambiente Construído, 2001, São Pedro: em Anais, p. 1.

NYEMEYER, M. L.; SLAMA, J. G. O Ruído na Cidade: Elementos do Ruído Urbano, In: Arquitetura: Pesquisa \& Projeto, Rio: Pró-Editores. UFRJ, 1998.

PLANO DIRETOR DE DESENVOLVIMENTO URBANO - II PDDU, Prefeitura Municipal de Passo Fundo - RS, 1984.

SMAM - Secretaria Municipal do Meio Ambiente, Prefeitura Municipal de Passo fundo, Dados de arquivo, 2001.

SMSU - Secretaria Municipal de Serviços Urbanos, Prefeitura Municipal de Passo Fundo, Dados de arquivo, 2005.

SOUSA, Denise da Silva de, Instrumentos de Gestão de poluição Sonora para a Sustentabilidade das Cidades Brasileiras, Tese de doutorado, COOPE/UFRJ, Rio de Janeiro, 2004.

WHO, Environmental Health Criteria 12 - Noise, World Health Organization, Genéve, 1980. 